\title{
LPS- induced inflammation exacerbates phospho- tau pathology in rTg4510 mice
}

\author{
Daniel C Lee ${ }^{1 *}$, Justin Rizer ${ }^{1}$, Maj-Linda B Selenica', Patrick Reid ${ }^{1}$, Clara Kraft², Amelia Johnson², Laura Blair², \\ Marcia N Gordon ${ }^{1}$, Chad A Dickey ${ }^{2}$, Dave Morgan ${ }^{1 *}$
}

\begin{abstract}
Inflammation and microglial activation are associated with Alzheimer's disease (AD) pathology. Somewhat surprisingly, injection of a prototypical inflammatory agent, lipopolysaccharide (LPS) into brains of amyloid precursor protein (APP) transgenic mice clears some of the pre-existing amyloid deposits. It is less well understood how brain inflammation modulates tau pathology in the absence of $A \beta$. These studies examined the role of LPS-induced inflammation on tau pathology. We used transgenic rTg4510 mice, which express the P301L mutation (4RON TauP301L) and initiate tau pathology between 3-5 months of age. First, we found an age-dependent increase in several markers of microglial activation as these rTg4510 mice aged and tau tangles accumulated. LPS injections into the frontal cortex and hippocampus induced significant activation of CD45 and arginase 1 in rTg4510 and non-transgenic mice. In addition, activation of YM1 by LPS was exaggerated in transgenic mice relative to nontransgenic animals. Expression of Ser199/202 and phospho-tau Ser396 was increased in rTg4510 mice that received LPS compared to vehicle injections. However, the numbers of silver-positive neurons, implying presence of more pre- and mature tangles, was not significantly affected by LPS administration. These data suggest that inflammatory stimuli can facilitate tau phosphorylation. Coupled with prior results demonstrating clearance of A $\beta$ by similar LPS injections, these results suggest that brain inflammation may have opposing effects on amyloid and tau pathology, possibly explaining the failures (to date) of anti-inflammatory therapies in AD patients.
\end{abstract}

\section{Background}

Tauopathies consist of intracellular accumulation of the microtubule-associated protein tau in the somatodendritic compartment associated with hyperphosphorylation and aggregation of the protein. Tau dysfunction can lead to neurodegeneration, motor dysfunction, and behavioral deficits in animal models that express mutated forms of the protein [1-4]. One of the most common tauopathies includes Alzheimer's disease (AD). Consequently, numerous studies targeting different components of the disease have been initiated to reduce tau pathology as well as amyloid- $\beta$. These include inhibition of kinases which phosphorylate tau, such as glycogen synthase kinase-3 $\beta$ (GSK3 $\beta$ ) [5-8], manipulating heat shock proteins [9-11], immunotherapy which targets the tau peptide and subsequently reduces $\mathrm{p}$-tau levels, $[12,13]$, and modifying $\mathrm{p}$-tau by manipulating the

\footnotetext{
*Correspondence: dlee1@health.usf.edu; scientist.dave@gmail.com 'Byrd Alzheimer's Institute, Department of Molecular Pharmacology and Physiology, University of South Florida, Tampa, FI 33612, USA Full list of author information is available at the end of the article
}

immune response [14]. Some reports indicate that pathological tau induces inflammation [15] and that inflammation modifies tau [16,17].

Inflammation arguably plays a significant role in the progression of AD pathology. The microglial activation state contributes to many of the ongoing debates, and it is believed that microglia can cause both beneficial and detrimental effects, depending on the microenvironment and cytokines involved. Recently, more attention has been paid to the functional status of microglia rather than generalized activation by generic markers. As more studies emerge identifying selective markers that represent different phenotypic activation states of microglia, an association of their role during disease pathology is being revealed. In the classical or M1 state, pro-inflammatory cytokines produce tissue damage and pathogen destruction, whereas the alternative activation state (M2) dampens this response and directs tissue repair and healing responses [18]. Some reports suggest that in chronic neurodegenerative diseases like AD, a hybrid activation state exists including markers of both M1 and
C Biomed Central 
M2 phenotypes [19]. Other reports argue that beginning stages of AD pathology in animal models of amyloid- $\beta$ deposition resemble an M2 state that switches to a more classical response with age [20]. It is possible the phenotypic state of microglia in response to amyloid- $\beta$ deposition influences certain aspects of tau pathology as well. Thus, it is important to identify the components of inflammation that promote vs. reduce tau pathology in order to design better therapeutic strategies which target the immune response.

In previous studies [21-25], intracranial LPS, which induces both M1 and M2 markers, activates microglia and reduces $A \beta$ pathology in APP transgenic models of amyloid deposition. This requires microglial activation and can be suppressed by dexamethasone administered systemically. Importantly, there is no indication for systemic inflammation in AD patients [26]. Herein, we similarly provoked central microglial activation by LPS to evaluate phospho-tau species and pathology in the rTg4510 mice. This model develops tangle pathology in the higher forebrain cortical layers and hippocampus coupled with cognitive deficits and neuronal loss $[1,4,27]$. To our knowledge, this is the first report showing that activation of inflammation in the brain exacerbates tau phosphorylation.

\section{Methods}

\section{Mouse breeding, tissue preparations, and animal} treatments

The rTg4510 mice, lines carrying the parental tau mutations and the tetracycline-controlled transactivator protein (tTA) were used [3]. For age-related microglia activation, brains were harvested from 1, 5, or 9 month old rTg4510 mice and their non-transgenic littermates. For lipopolysaccharide (LPS) studies, male and female mice were aged 4.5 months and a volume of $2 \mu$ of LPS (5 $\mu \mathrm{g} / \mu \mathrm{l}$; Salmonella abortus equii, Sigma-Aldrich, St. Louis MO) in was unilaterally injected into the hippocampus and anterior cortex (frontal cortex area3) of rTg4510 and non-transgenic littermates. Stereotaxic coordinates from bregma were $+1.7 \mathrm{~mm}$ anteroposterior, $-2.2 \mathrm{~mm}$ lateral and $-2.5 \mathrm{~mm}$ vertical for frontal cortex, and, $-2.7 \mathrm{~mm}$ anteroposterior, $-2.7 \mathrm{~mm}$ lateral and -3.0 $\mathrm{mm}$ vertical for hippocampus. The solution was dispensed at a constant rate of $0.5 \mu \mathrm{l} / \mathrm{min}$. Seven days post injection; mice were weighed and overdosed with 100 $\mathrm{mg} / \mathrm{kg}$ of pentobarbital. Mice were then perfused intracardially with $25 \mathrm{ml}$ of $0.9 \%$ saline. The brain was removed, and immersion fixed in $4 \%$ paraformaldehyde in $100 \mathrm{mM} \mathrm{PO}_{4}$ buffer ( $\mathrm{pH}$ 7.4) for 24 hours. The tissue was cryoprotected in a series of $10 \%, 20 \%$ and $30 \%$ sucrose solutions. Horizontal sections were cut at $25 \mu \mathrm{m}$ using a sliding microtome and stored at $4^{\circ} \mathrm{C}$ in
Dulbecco's phosphate buffered saline containing $100 \mathrm{mM}$ sodium azide for immunohistochemistry.

\section{Immunohistochemistry and silver stain}

Immunohistochemistry was performed on free floating sections as described in detail previously [28]. Sections were incubated with primary antibodies rat anti-mouse CD45 (1:3000) (Serotec, Raleigh, NC), rat anti-major histocompatibility complex -II (1:5000) (MHCII; BD Pharmigen), rabbit anti-mouse chitinase 3-like-3 (1:3000) (YM1; StemCell Technologies, Vancouver, Canada), chicken anti-arginase 1 (1:50,000)(generous gift from Dr. S.M. Morris)[29], rabbit anti-human phosphotau ser199/202 (1:65,000)(Anaspec, Fremont, CA), rabbit anti-human phospho-tau ser396 (1:3000) (Anaspec, Fremont, CA), or rabbit anti- human full length-tau (1:3000) (H-150, sc-5587, Santa Cruz Biotechnology, Santa Cruz, CA), AT8 (1:5000) (Thermo Scientific, Rockford, IL overnight at $4^{\circ} \mathrm{C}$, then incubated in the appropriate biotinylated secondary antibody (VectorLabs, Burlingame, CA) for $2 \mathrm{~h}$ followed by a $1 \mathrm{hr}$ incubation in ABC (Vector Labs, Birlingame, CA). Color development was performed using 0.05\% 3, 3'-diaminobenzidine (DAB; Sigma, St. Louis, MO) enhanced with $0.5 \%$ nickelous ammonium sulfate (J. T. Baker Chemical Company, Phillipsburg, NJ). For silver staining, a series of sections stained using Gallyas silver stain method [2]. Briefly, sections were fixed in $4 \%$ paraformaldehyde in $100 \mathrm{mM} \mathrm{PO}_{4}$ buffer ( $\mathrm{pH} \mathrm{7.4)}$ for 24 hours, horizontally sectioned at $25 \mu \mathrm{m}$ thickness, and stored at $4^{\circ} \mathrm{C}$ in Dulbecco's phosphate buffered saline containing $100 \mathrm{mM}$ sodium azide. Free floating sections were mounted on slides and processed together using Gallyas silver stain method with the omission of a counter stain for quantitative analysis. It should be noted for time courses and LPS studies that all tissue sections for each immunohistochemical stain and the Gallyas silver stain that was analyzed together were processed together at the same time under the same conditions.

\section{Immunofluorescence}

Immunohistochemistry was performed on free floating sections as previously describe above with slight modifications to primary antibody concentrations. Sections were incubated with primary antibodies rat anti-mouse CD45 (1:1000), rabbit anti-human phospho-tau ser199/ 202 (1:10,000), rabbit anti-mouse chitinase 3-like-3 (YM1) (1:1000), rabbit anti-human phospho-tau ser396 (1:1000), rabbit anti- human full length-tau (1:2000) (H-150), biotinylated AT8 (1:5000) (Thermo Scientific, Rockford, IL) overnight at $4^{\circ} \mathrm{C}$, washed and incubated with the appropriate secondary Alexa Fluor antibodies for $2 \mathrm{~h}$ (Invitrogen); goat anti-rabbit Alexa 488, goat 
anti-rat Alexa 488, Streptavidin Alexa 594, donkey antichicken Alexa 488, goat anti-rabbit Alexa 594. Sections were mounted on slides with Vectashield, (Vector Labs, Burlingame, CA).

\section{Image analysis quantification and statistics}

Immunohistochemical staining was quantified with Image Pro Plus (Media Cybernetics, Silver Spring, MD) image software. Positively labeled microglia or tau positive neurons were segmented using RGB intensity. Each brain section was imaged at $100 \times$ magnification in the anterior cortex centered on the injection site (frontal cortex, area 3 ), the CA1 or CA3 region of the hippocampus, and entorhinal cortex (caudomedial). Data were obtained as a percent area of the image field that was positively stained by immunochemical or histochemical reaction product. Some sections were digitized on the Zeiss Mirax slide scanner. All values (8 sections) obtained from a single mouse were then averaged to represent a single value for each brain region. Statistical analysis was performed using 2-way ANOVA (Age and Treatment), followed by Fisher's LSD post hoc means comparison test with $\mathrm{p}$ values of $<0.05$ considered significant using Stat View software version 5.0 (SAS Institute Inc, Cary NC). Graphs were generated using GraphPad Prism 4.0 (La Jolla, CA).

\section{Results}

\section{Age-related CD45 activation in $\mathrm{rTg} 4510$ mice}

Previous work has characterized age-related accumulation of various phospho-tau species in forebrain areas and hippocampus of rTg4510 mice [30]. A cross-sectional analysis showed accumulation of insoluble tau species as early as 5.5 months of age. Herein, we evaluated CD45, MHCII and an alternative activation marker, YM1, as markers of microglial activation at 1, 5, and 9 months of age in rTg4510 mice and their non-transgenic littermates. Representative images of anterior cerebral cortex and hippocampus after immunostaining for CD45 are presented in Fig. 1. Neither nontransgenic nor rTg4510 display appreciable staining at one month of age in either cortex (Fig. 1A, B) or hippocampus (Fig. $1 \mathrm{H}, \mathrm{I})$. More staining is apparent in sections from older mice (Fig. 1C-F, J-K). Significant activation of CD45 was observed at 9 months in the anterior cortex (Fig.1G) and hippocampus (Fig.1N) compared with age-matched, nontransgenic littermates. Furthermore, CD45 expression of 9 month old rTg4510 mice was significantly greater than observed in either 1 or 5 month old mice in the hippocampus (Fig. 1N) and greater than 1 month old mice in cortex (Fig.1G).

We also examined the microglial markers MHC II and YM-1 as a function of age in $\operatorname{rTg} 4510$ mice and their nontransgenic littermates. However, although occasional microglia were positive for MHC II, these were only observed in 9 month old rTg4510 mice (data not shown). We failed to detect any positive YM1 microglia at any age. These data show that age-related accumulation of pathological tau induces CD45 activation in the forebrain of rTg4510 mice.

\section{LPS induced CD45, YM1 and arginase-1 in rTg4510 mice}

Previous data show that certain inflammatory events modify the pathology in animal models which deposit amyloid [24]. LPS-induced microglial activation reduces amyloid burdens in the brains of APP Tg2576 mice within one week [23]. We used a similar approach to evaluate tau pathology 1 week following intracranial LPS administration into anterior cortex and hippocampus. LPS injections dramatically induced CD45 activation on the ipsilateral side of the anterior cortex (Fig. 2A-E), hippocampus (Fig. 2F-J), and entorhinal cortex (Fig. 2KO) compared to vehicle- treated mice in both rTg4510 mice and nontransgenic littermates. Furthermore, significant LPS-induced CD45 activation was also observed on the contralateral side of the cortex and hippocampus compared to vehicle-treated mice (Table 1), although to a lesser extent. The magnitude of LPS-induced CD45 activation was similar in $\mathrm{rTg} 4510$ and non-transgenic mice.

LPS also significantly increased the alternative activation marker YM1 in the ipsilateral hemisphere of the anterior cortex (Fig. 3A-E), hippocampus (Fig. 3F-J), and entorhinal cortex (Fig. 3K-O) compared to vehicle-treated groups in both rTg4510 mice and in nontransgenic littermates. However, unlike CD45 activation, YM1 induction was significantly greater in $\mathrm{rTg} 4510$ mice compared to non-transgenic mice. Furthermore, YM1 activation also increased on the contra-lateral hemisphere following LPS (Table 1) and this response was also augmented in the hippocampus and entorhinal cortex of rTg4510 mice compared to nontransgenic littermates.

We also evaluated arginase 1, typically associated with alternative activation. LPS induced robust expression of arginase 1 staining on the ipsilateral side of the anterior cortex (Fig 4A-E), hippocampus (Fig. 4F-J), and entorhinal cortex (Fig. 4K-O) compared to vehicle-treated mice. There was no difference in the size of arginase 1 induction between rTg4510 and nontransgenic mice.

\section{LPS-Induced inflammation exacerbates phospho-tau pathology}

To evaluate the effects of LPS-induced inflammation on tau pathology, we measured pre-tangle pathology by phospho-tau staining, in addition to mature insoluble tangle pathology by Gallyas silver stain. At 5 months, detectable levels of tau phosphorylated at epitope ser199/202 were 


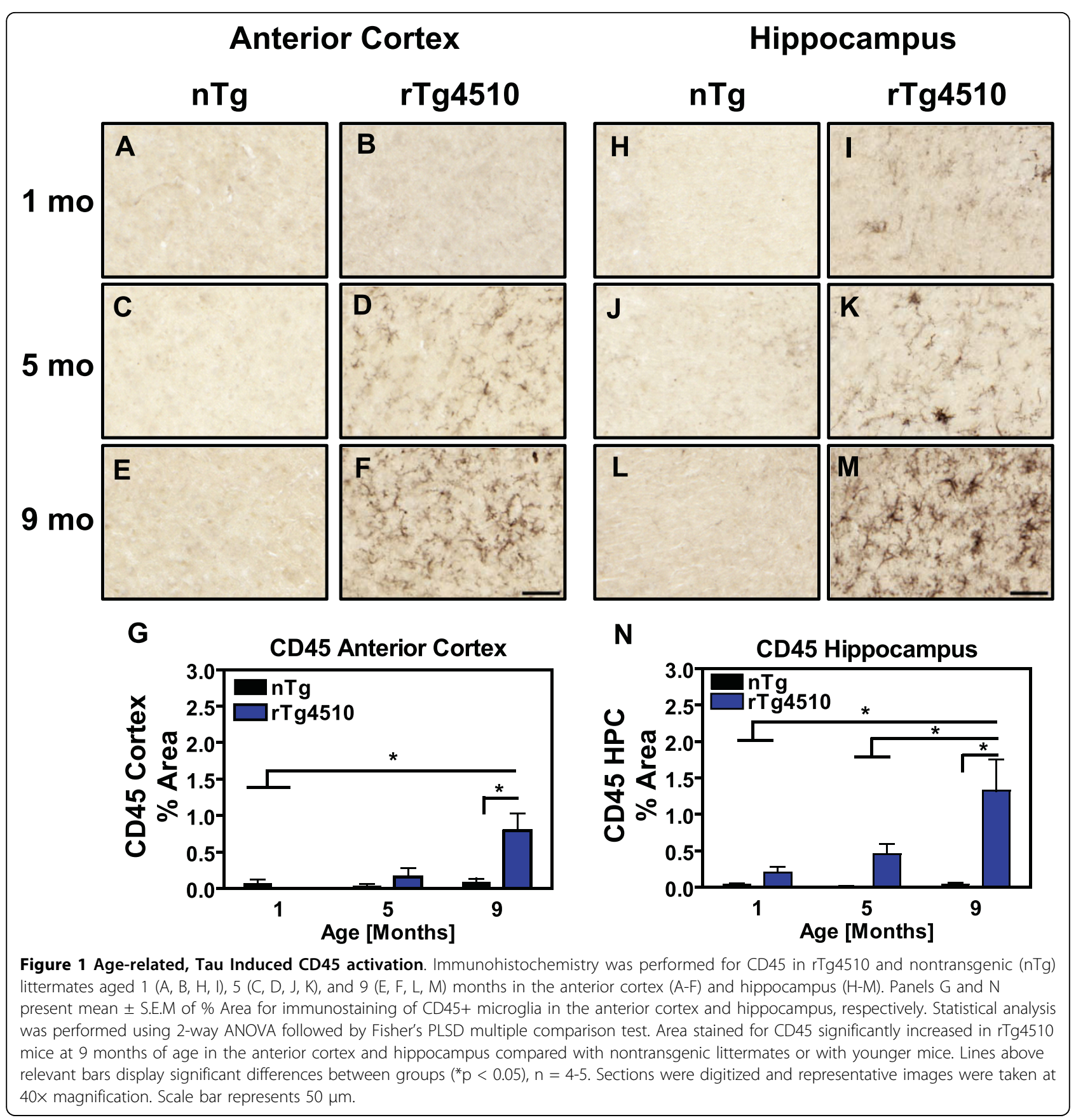

observed in vehicle- treated rTg4510 mice (Fig. 5B, G, L). LPS significantly increased p-tau ser199/202 in the anterior cortex (Fig. 5A-E) and entorhinal cortex (Fig. 5K-O) in $\mathrm{rTg} 4510$ mice compared to the vehicle- treated rTg4510 mice. Although the mean \% area for staining of phospho-tau ser199/202 in the hippocampus following LPS administration showed an elevated trend (Fig. 5F-J), it failed to reach statistical significance. Likewise, phosphotau epitope ser396 was observed in vehicle-treated mice along axonal processes and in perinuclear regions, yet
LPS-induced inflammation further increased phospho-tau ser396 immunoreactivity in the cortex (Fig. 6A-E), hippocampus (CA1; Fig. 6F-J), and entorhinal cortex (Fig. 6K-O) compared to rTg4510 mice treated with vehicle. Neither phospho-tau species (tau ser199/202 or ser396) was detectable at the immunohistochemical level in nontransgenic mice which received vehicle or LPS administration.

To identify the impact of LPS-induced inflammation on pre-tangle and mature tau pathology, we measured Gallyas silver staining in $\mathrm{rTg} 4510$ mice and nontransgenic 


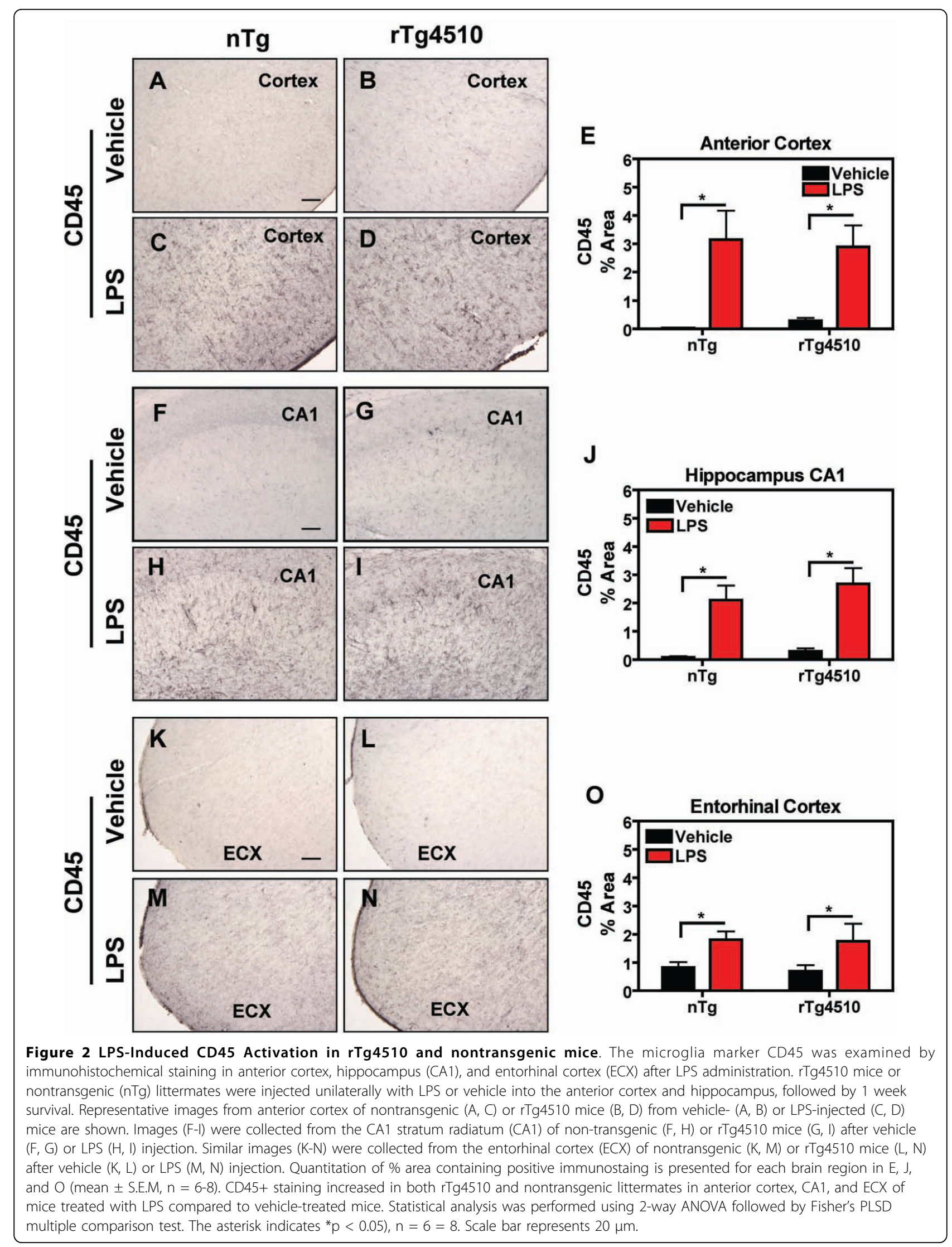


Table 1 LPS-Induced Microglia Activation on Contralateral Hemisphere

\begin{tabular}{cccccc}
\hline & & \multicolumn{2}{c}{ rTg4510 } \\
\hline CD45 & Region & Vehicle & LPS & Vehicle & LPS \\
\hline & CX & $0.008 \pm 0.003$ & $0.397 \pm 0.162$ & $0.161 \pm 0.110$ & $0.778 \pm 0.270^{\mathrm{a}}$ \\
& CA1 & $0.027 \pm 0.015$ & $0.410 \pm 0.100$ & $0.250 \pm 0.151$ & $0.489 \pm 0.154^{\mathrm{a}}$ \\
& CA3 & $0.061 \pm 0.020$ & $2.325 \pm 0.431$ & $0.112 \pm 0.024$ & $1.310 \pm 0.527^{\mathrm{a}}$ \\
& ECX & $0.530 \pm 0.093$ & $0.920 \pm 0.182$ & $0.541 \pm 0.118$ & $0.875 \pm 0.409$ \\
\hline YM1 & Region & Vehicle & LPS & Vehicle & LPS \\
\hline CX & $0.001 \pm 0.001$ & $0.024 \pm 0.005$ & $0.001 \pm 0.001$ & $0.018 \pm 0.006^{\mathrm{a}}$ \\
& CA1 & $0.003 \pm 0.002$ & $0.025 \pm 0.007$ & $0.002 \pm 0.001$ & $0.100 \pm 0.036^{\mathrm{a}, \mathrm{b}}$ \\
& CA3 & $0.001 \pm 0.001$ & $0.049 \pm 0.008$ & $0.004 \pm 0.002$ & $0.154 \pm 0.049^{\mathrm{a}, \mathrm{b}}$ \\
& ECX & $0.005 \pm 0.002$ & $0.080 \pm 0.020$ & $0.001 \pm 0.001$ & $0.586 \pm 0.189^{\mathrm{a}, \mathrm{b}}$ \\
& Region & Vehicle & LPS & Vehicle & LPS \\
\hline & CX & $0.008 \pm 0.001$ & $0.012 \pm 0.005$ & $0.005 \pm 0.001$ & $0.008 \pm 0.002$ \\
& CA1 & $0.014 \pm 0.002$ & $0.020 \pm 0.007$ & $0.008 \pm 0.003$ & $0.013 \pm 0.003$ \\
& CA3 & $0.017 \pm 0.002$ & $0.044 \pm 0.024$ & $0.007 \pm 0.002$ & $0.047 \pm 0.023$ \\
\hline
\end{tabular}

For each marker, 2-way ANOVA follwed by Fisher's LSD Analysis for genotype and treatment were performed. ap < 0.05, Vehicle v.s LPS; bp < 0.05, nTg v.s rTg4510 mice. CD45; microglial activation marker, YM1; microglial alternative activation marker, Arginase-1; alternative activation marker. CX; anterior cortex, CA1 and CA3; hippocampus CA1 and CA3 respectfully, ECX; entorhinal cortex. $n=5-8$.

littermates. Vehicle-treated rTg4510 mice at 5 months of age had small but measurable amounts of Gallyas silver positive neurons. Following LPS administration no significant increases or decreases were observed in the anterior cortex (Fig. 7A-E), hippocampus (Fig. 7F-J), or entorhinal cortex (Fig. 7K-O) of rTg4510 mice. This suggests that acute LPS-induced microglial activation impacts tau phosphorylation but, at least within the first week, does not affect the pre- and mature tangles as determined by Gallyas stain.

We also evaluated full-length tau in the anterior cortex, hippocampus, and entorhinal cortex by immunohistochemistry (Fig. 8). As observed with the silver stain, tau (H-150) antibody failed to recognize endogenous mouse tau in nontransgenic mice, under these staining conditions at the immunohistochemisrty level (Fig.8AF). However, detectable levels were observed in rTg4510 mice (Fig. 8G-H) in cortical regions and hippocampus, but were not increased by LPS treatment (Fig. 8M) as was the case for phospho-tau markers potentially due to recognition of multiple isoforms.

\section{Double labeling of phospho-tau and microglia}

To further identify the relationship between phosphotau expressing cells and microglia, we performed double labeling studies on rTg4510 mice following LPS injections. Arginase- 1 expression was observed in rod and amoeboid-like with some branching cells (Fig. 9A-C) and failed to co-localized with cells stained for phosphotau Ser396. YM1 positive cells were highly branched and also failed to co-localize with cells stained with the AT8 (phospho-tau Ser202/Thr205) (Fig.9D-F), however several YM1 positive microglia were clustered around AT8 positive neurons. Furthermore, CD45 positive cells displayed various cell morphologies from highly branched to amoeboid/rod and macrophage-like. In general, CD45 activation increased around tau laden areas (phospho-tau Ser199/202 and total tau) such as hippocampus (Fig. 9G-L). Overall, there was rarely cell to cell association and microglia failed to clearly co-localize with phospho-tau positive neurons.

\section{Discussion}

In this study, we show that the phosphorylated tau species previously characterized in the $\mathrm{rTg} 4510$ mice [30] are associated with age-related microglial activation as measured by CD45 Further activation of microglia by LPS enhances tau phosphorylation. Prior work [30], demonstrated that young mice possess the ability to clear soluble phospho-tau species, showing reductions in these markers between 1 and 3 months. However, by 5.5 months, insoluble tau aggregates appear in parallel with accumulation of a $64 \mathrm{kD}$ soluble tau species. Thus, microglial activation begins at this age when soluble and insoluble tau species are present. When microglial activation is provoked by LPS challenge at this point, there are clear increases in the phosphorylation of tau. Previous studies showed that LPS-induced microglial activation in APP mice clears amyloid- $\beta$ pathology in the CNS as early as 3 days following intracranial injection [21-24]. Using this same paradigm, LPS-induced microglial activation in rTg4510 mice exacerbates pre-tangle pathology as visualized by phospho-tau staining. This highlights the need to include mouse models of tau 


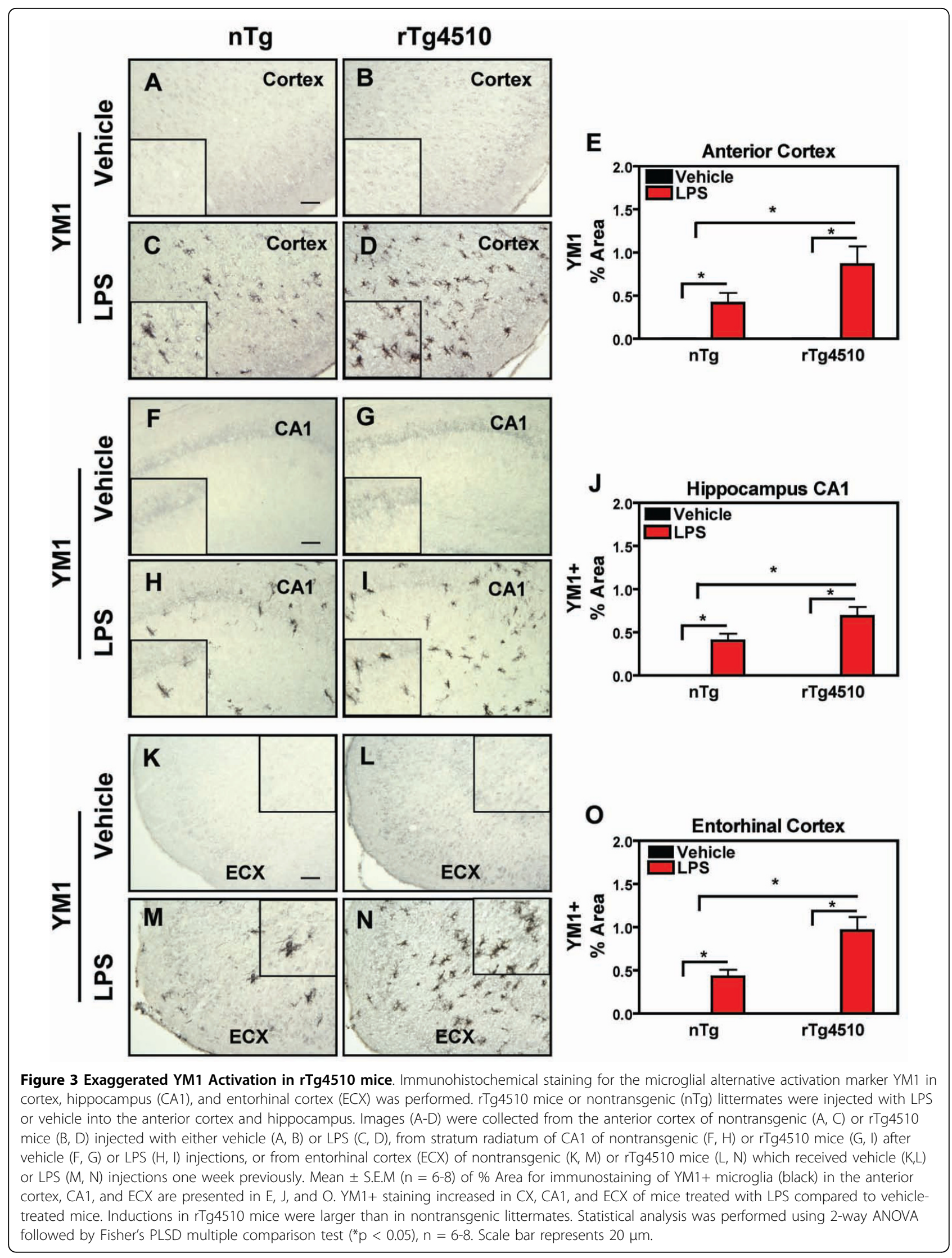




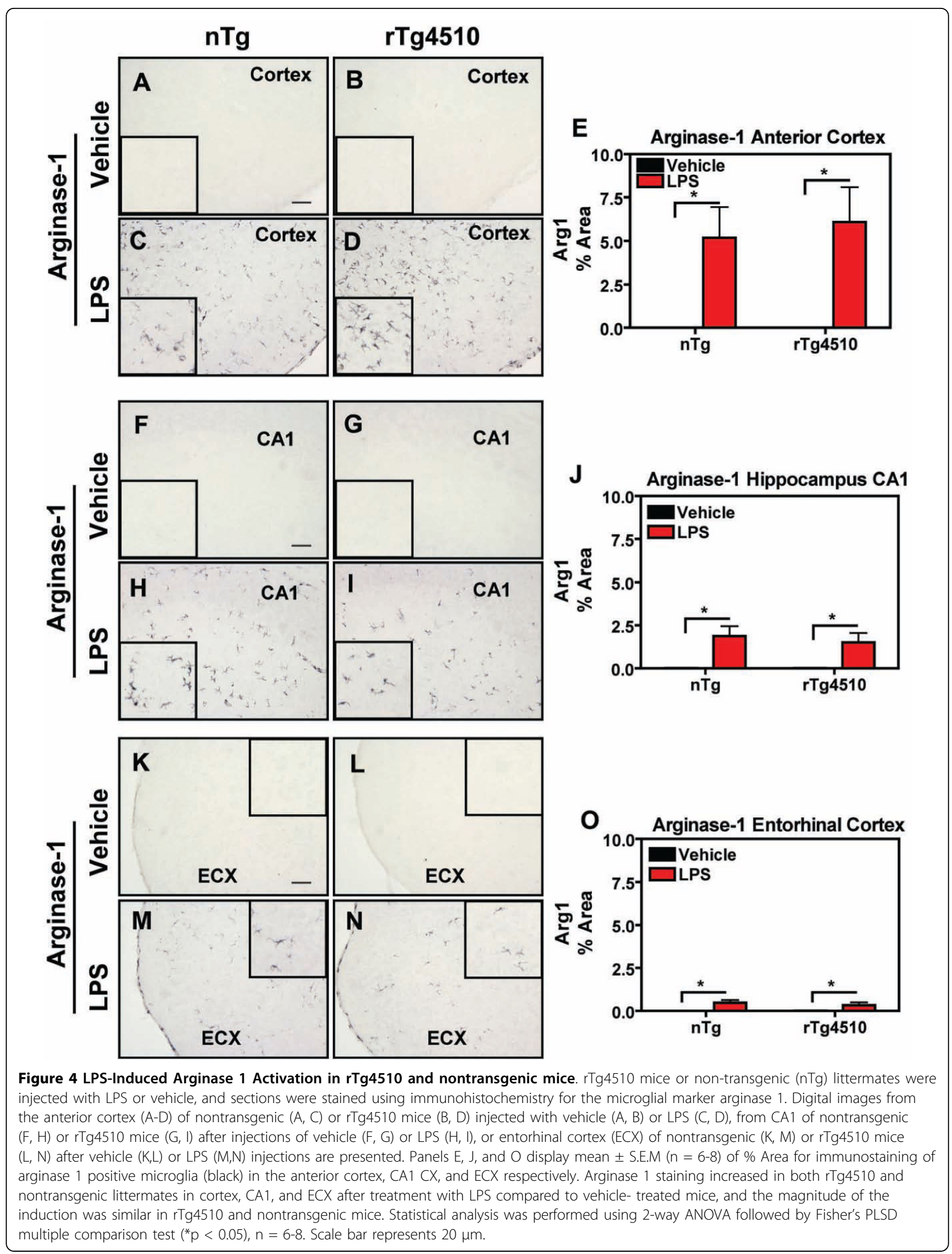




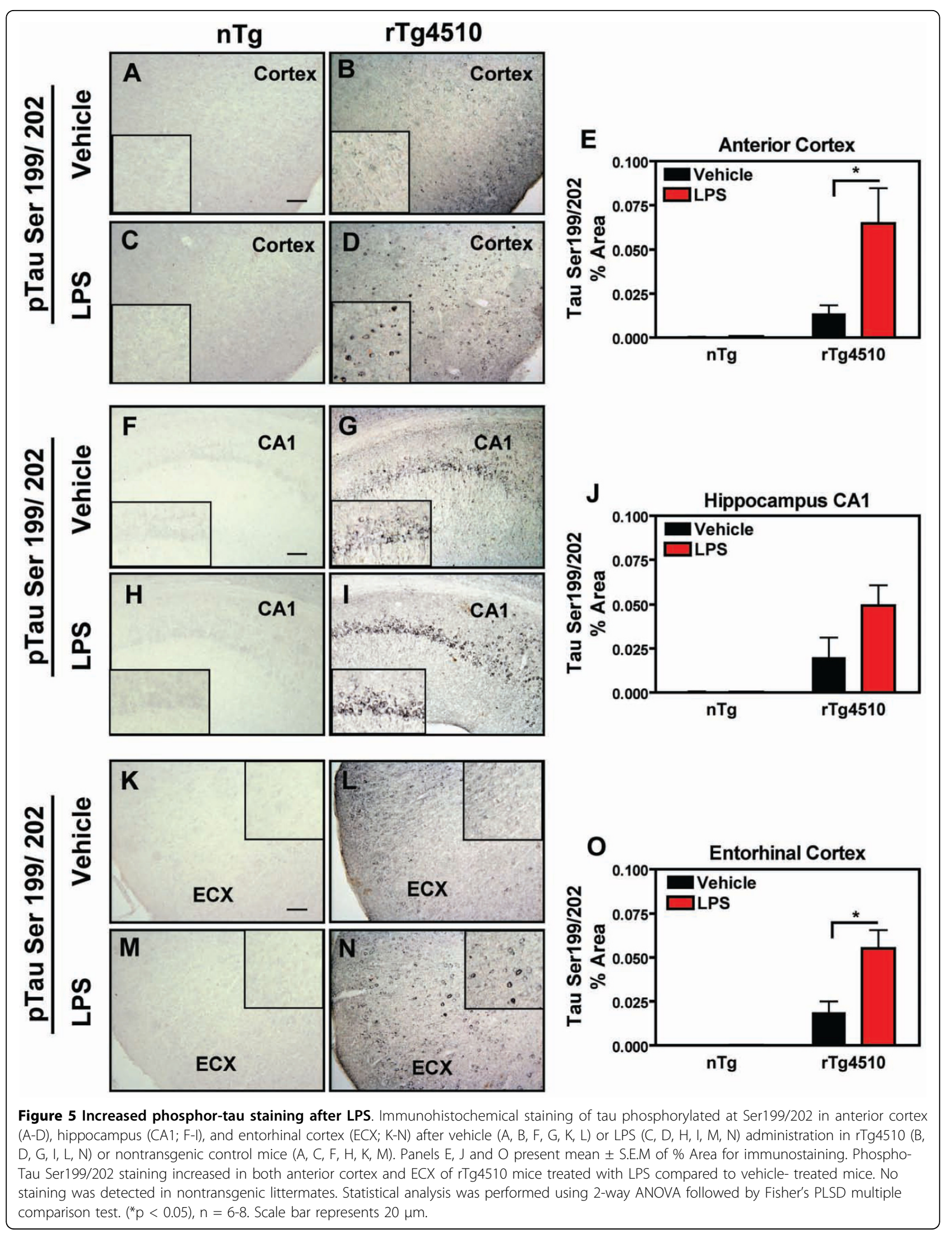




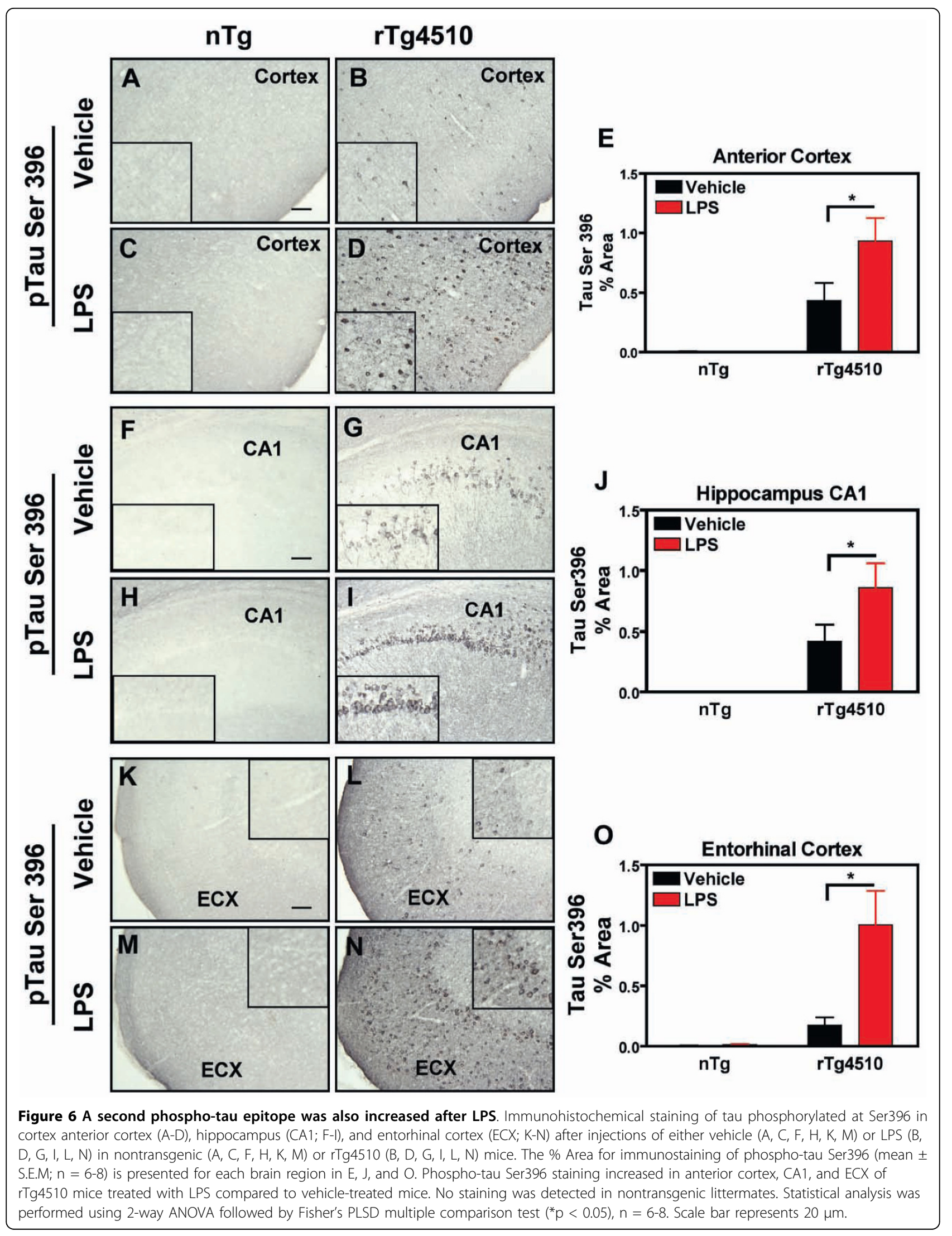




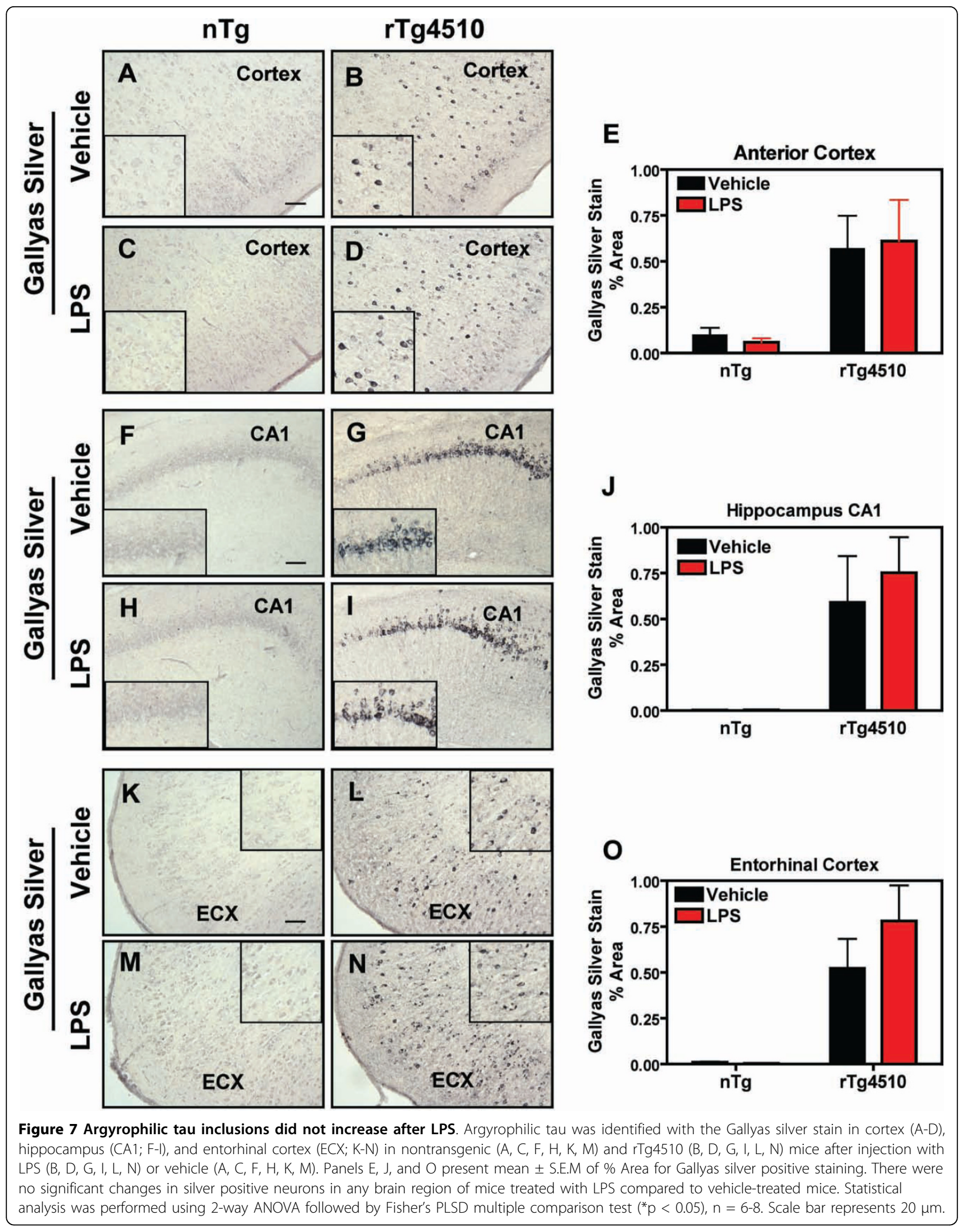




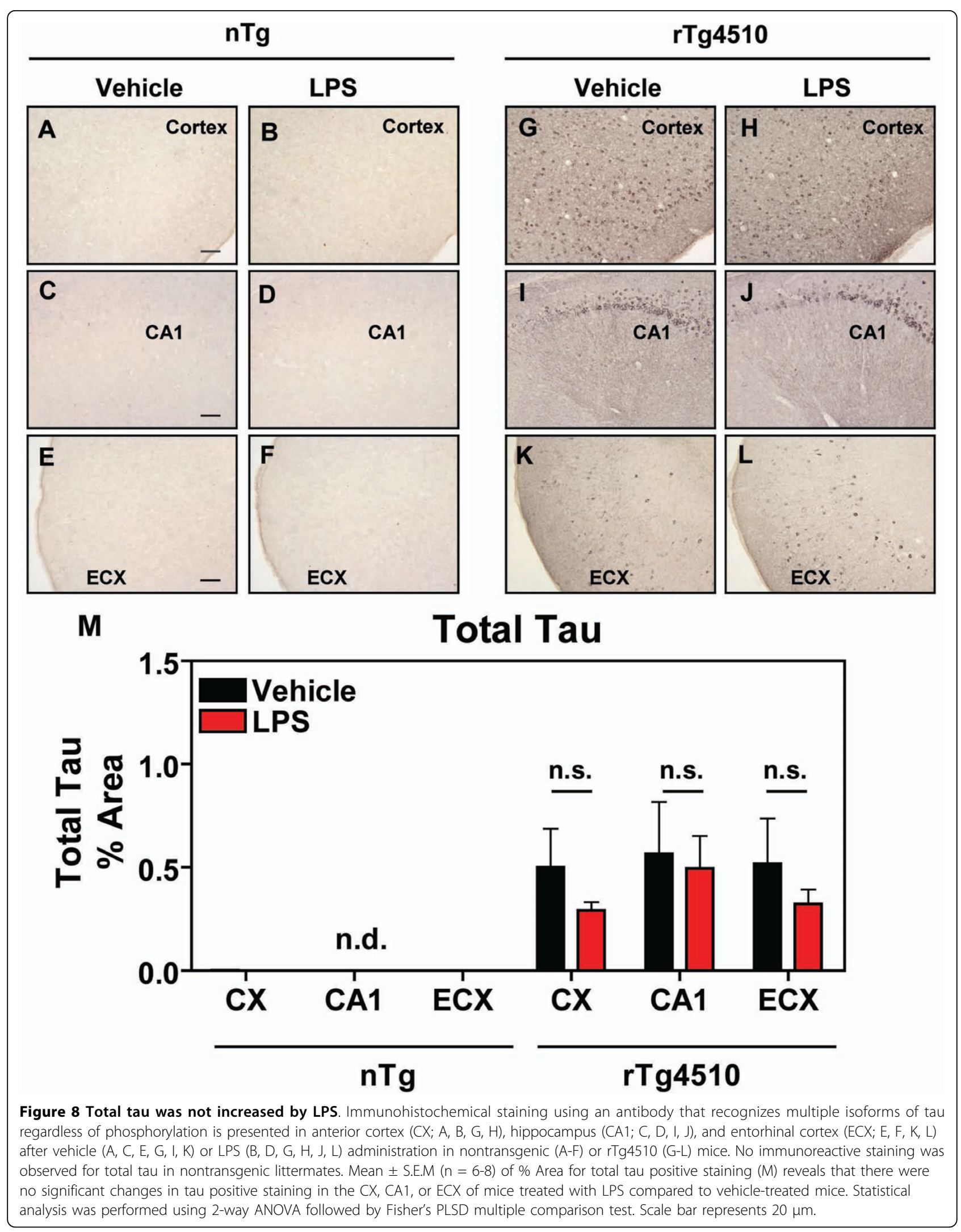




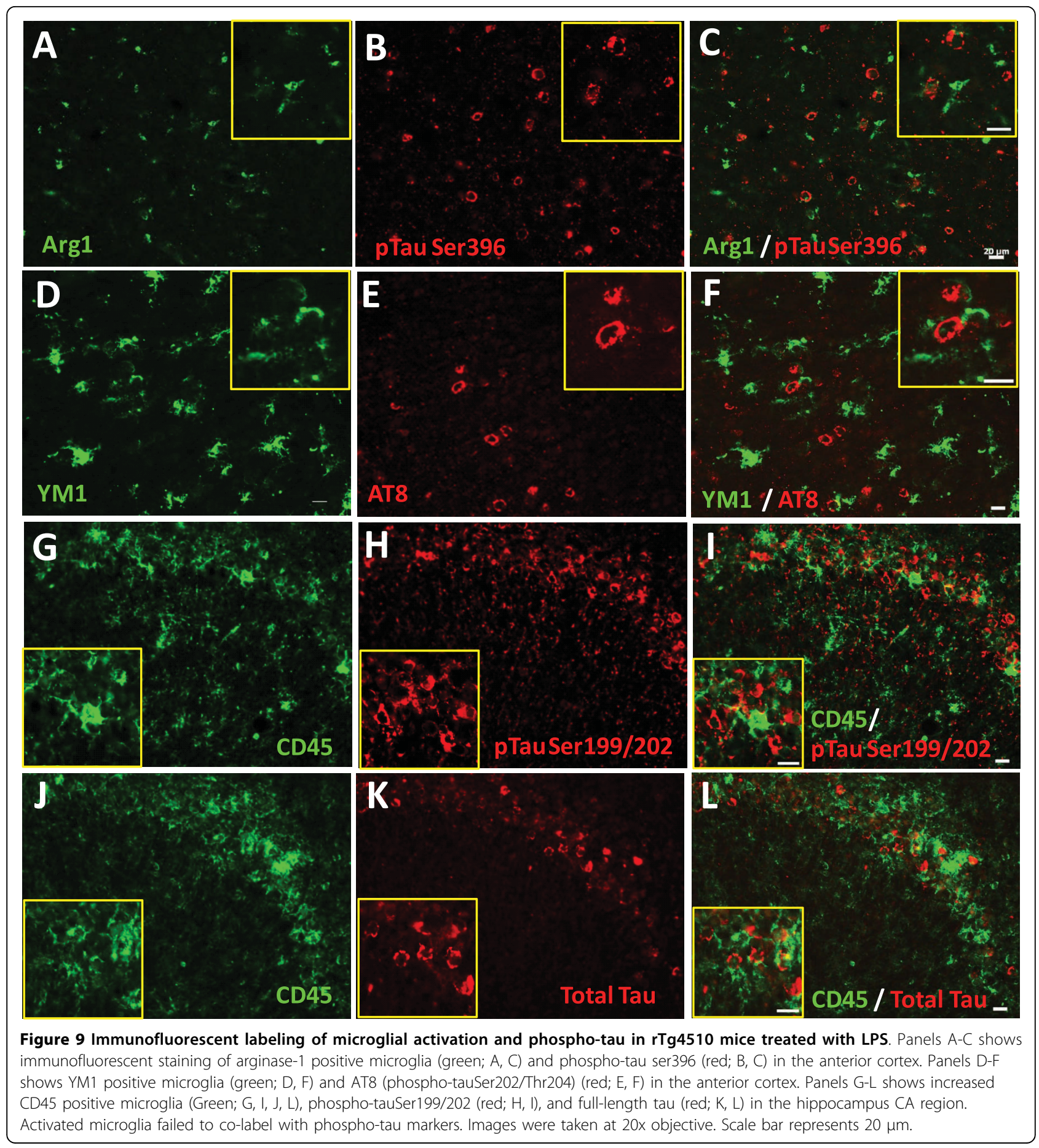

pathology as well as models of amyloid pathology when assessing the impact of potential treatments for translation in to clinical trials in Alzheimer cases.

Another previous study using a 3xTg-AD model (harboring $\mathrm{APP}_{\mathrm{K} 670 \mathrm{~N} ; \mathrm{M} 671 \mathrm{~L}}$, $\mathrm{PS}_{\mathrm{M} 146 \mathrm{~V}}$, and $\mathrm{Tau}_{\mathrm{p} 301 \mathrm{~L}}$ mutations) showed no changes in APP processing after 6 weeks of peripheral administration of LPS. However, phosphorylation of tau at specific sites (AT-180, p231/ 235; AT8, p202/205; but not PHF-1, p396/404) was increased within the hippocampus, in a cyclin kinase 5dependent mechanism [16]. Another pro-inflammatory stimulus, interleukin- $1 \beta$, also resulted in microglial activation and tau phosphorylation in cortical neurons [17]. Herein, we show that acute activation of microglia by 
LPS increased phospho-tau staining within one week, not only in the hippocampus and anterior cortex, but also in other tau-laden areas that were not injected including entorhinal cortex. Although the level of microglial activation also increased in the entorhinal cortex to a lesser extent than that of the hippocampus and anterior cortex, the increased phospho-tau species observed distal to the injection site is conceivable from previous findings of systemic inflammation and CNS effects on phospho-tau [16] and supports the potential role for diffusible ligands and cytokines and their impact on tau pathology. Although these data suggest that acute inflammatory conditions may accelerate the course of neurodegenerative tauopathies or AD, other models of low level chronic neuroinflammation should be explored in a similar context [31].

With normal aging up to 9 months, CD45 positive microglia increased in parallel with tau pathology, yet the alternative activation marker YM1 was not detected at the protein level by immunohistochemistry. However, upon LPS challenge, YM1 was elevated to a significantly greater level in rTg4510 mice with pre-existing tau than in nontransgenic mice. Thus, not only does microglia activation appear to influence tau pathology but tau pathology appears to impact the phenotypic responses of microglia as well.

It has yet to be determined whether this exaggerated YM1 activation occurred in response to insoluble vs. soluble tau species or even other specific tau forms such as truncated tau. A recent report showed that human misfolded, truncated tau protein promoted the up-regulation of immune molecules in microglia/macrophages and caused the influx of antigen-presenting cells from blood into the CNS of transgenic rats [15]. It is also unknown whether YM1 influences the pathological state of tau, however recent reports show YM1 protein expression localizes around the amyloid plaques of APP/ PS1 transgenic mice, and AD brains contain increased YM1 mRNA compared to normal, aged -matched control brains $[19,20]$. This implies that amyloid pathology can increase YM1 expression. YM1, whose function is poorly understood, exists as a secretory protein transiently expressed in microglia/macrophages during hematopoiesis [32], during parasitic infection or after interleukin-4/interleukin-13 cytokine stimulation [33]. YM1 shows specific binding affinity to glucosamine, galactosamine, and heparin sulfate, which has been hypothesized as a mechanism for shielding or maintaining macrophage integrity during parasitic infection $[32,34]$. Interestingly, heparin sulfate and sulfated glycosaminoglycans prevent tau from binding to microtubules, promote microtubule disassembly, and stimulate tau phosphorylation by several kinases [35]. Although,
LPS induced inflammation and tau pathology seems to influence the expression of YM1, it is unclear if up-regulation of YM1 by cytokines or amyloid- $\beta$ deposition directly impacts the tau pathology.

Gene delivery of the pro-inflammatory cytokine tumor necrosis factor alpha (TNF- $\alpha$ ) into the CNS of 3xTg-AD mice resulted in accumulation of both $A \beta 42$ and phospho-tau species [36]. Conversely, chronic ibuprofen treatment in 3xTg-AD mice reduced oligomeric amyloid- $\beta$, hyperphosphorylated tau, and improved memory deficits [37]. However, overexpression of the pro-inflammatory and M1-stimulating cytokine, interferon gamma, using AAV resulted in differential effects on amyloid- $\beta$ and phospho-tau[14]. The authors observed increased levels of amyloid- $\beta$ but reduced levels of phospho-tau, contradicting results with INF- $\gamma$ in 3xTg-AD mice [14]. Given the evidence that the amyloid deposition drives tau pathology in this 3xTg-AD model [12], it is unclear whether direct effects on tau or indirect effects on amyloid are responsible for changes in tau pathology.

Transgene regulated over-expression of the M1-stimulating cytokine, interleukin-1 in APP mice caused reductions in amyloid pathology [38]. Similarly, AAVmediated over-expression of interleukin-6 in TgCRND8 and Tg2576 mice elicited massive gliosis and reductions in amyloid- $\beta$ pathology [39]. The microglial phenotype in these mice included increased YM1, but not other M2 activation markers such as arginase 1. In our study, we observed a tau transgene facilitation of YM1 induction following LPS in both hemispheres, but this effect was not evident for arginase 1activation, another putative M2 activation marker. This raises questions regarding how particular M2 activation state markers are regulated by proinflammatory stimuli and why RNA and protein levels of YM1 are increased in AD patients and animal models of amyloid deposition, which are typically considered to be associated with a proinflammatory (M1) cytokine environment $[19,20]$. These observations are important in considering the ultimate goals for therapeutic tuning of the microglial phenotype in order to reduce amyloid and/or tau pathology. Some dichotomous effects in the different transgenic models and therapeutic treatments make interpretations and potential translation to $\mathrm{AD}$ challenging. These results suggest a more complex set of microglia phenotypes than the dipolar M1/M2 characterization, and suggest that the clearance of amyloid pathology and tau pathology may be mediated by distinct activation subtypes.

\section{Abbreviations}

AD: Alzheimer's disease; LPS: lipopolysaccharide, APP: amyloid precursor protein; AAV: adeno-associated virus 


\section{Acknowledgements}

The authors would like to thank Dr. Sidney M. Morris Jr. (University of Pittsburg School of Medicine) for gifting the arginase-1 antibody and reviewing the final manuscript.

\section{Author details}

'Byrd Alzheimer's Institute, Department of Molecular Pharmacology and Physiology, University of South Florida, Tampa, FI 33612, USA. ${ }^{2}$ Byrd Alzheimer's Institute, Department of Molecular Medicine, University of South Florida, Tampa, Fl 33612, USA.

\section{Authors' contributions}

DCL led the study and involved in all aspects from surgeries to analysis of data, and manuscript preparation. JR participated in surgeries, immunohistochemical staining, and data acquisition. MS and PR participated in tissue preparation, immunohistochemical/fluorescent staining and data acquisition, generation of figures. CK, AJ, and LB performed Gallyas Silver staining and maintained animal husbandry, breeding, and genotyping of mice and data collection. CD, MG, DM were involved in overall conception, secured funding for the project, experimental design, manuscript preparation and analysis. All authors have read and approved the final manuscript.

\section{Competing interests}

The authors declare that they have no competing interests.

Received: 25 March 2010 Accepted: 16 September 2010 Published: 16 September 2010

\section{References}

1. Berger Z, Roder H, Hanna A, Carlson A, Rangachari V, Yue M, Wszolek Z, Ashe K, Knight J, Dickson D, et al: Accumulation of pathological tau species and memory loss in a conditional model of tauopathy. J Neurosci 2007, 27:3650-3662.

2. Lewis J, McGowan E, Rockwood J, Melrose H, Nacharaju P, Van Slegtenhorst M, Gwinn-Hardy K, Paul Murphy M, Baker M, Yu X, et al: Neurofibrillary tangles, amyotrophy and progressive motor disturbance in mice expressing mutant (P301L) tau protein. Nat Genet 2000, 25:402-405.

3. Santacruz K, Lewis J, Spires T, Paulson J, Kotilinek L, Ingelsson M, Guimaraes A, DeTure M, Ramsden M, McGowan E, et al: Tau suppression in a neurodegenerative mouse model improves memory function. Science 2005, 309:476-481.

4. Ramsden M, Kotilinek L, Forster C, Paulson J, McGowan E, SantaCruz K, Guimaraes A, Yue M, Lewis J, Carlson G, et al: Age-dependent neurofibrillary tangle formation, neuron loss, and memory impairment in a mouse model of human tauopathy (P301L). J Neurosci 2005, 25:10637-10647.

5. Noble W, Planel E, Zehr C, Olm V, Meyerson J, Suleman F, Gaynor K Wang L, LaFrancois J, Feinstein B, et al: Inhibition of glycogen synthase kinase- 3 by lithium correlates with reduced tauopathy and degeneration in vivo. Proc Natl Acad Sci USA 2005, 102:6990-6995.

6. Leclerc S, Garnier M, Hoessel R, Marko D, Bibb JA, Snyder GL, Greengard P, Biernat J, Wu YZ, Mandelkow EM, et al: Indirubins inhibit glycogen synthase kinase-3 beta and CDK5/p25, two protein kinases involved in abnormal tau phosphorylation in Alzheimer's disease. A property common to most cyclin-dependent kinase inhibitors? J Biol Chem 2001 276:251-260.

7. Jope RS: Lithium and GSK-3: one inhibitor, two inhibitory actions, multiple outcomes. Trends Pharmacol Sci 2003, 24:441-443.

8. Sereno L, Coma M, Rodriguez M, Sanchez-Ferrer P, Sanchez MB, Gich I, Agullo JM, Perez M, Avila J, Guardia-Laguarta C, et al: A novel GSK-3beta inhibitor reduces Alzheimer's pathology and rescues neuronal loss in vivo. Neurobiol Dis 2009, 35:359-367.

9. Jinwal UK, Miyata Y, Koren J, Jones JR, Trotter JH, Chang L, O'Leary J, Morgan D, Lee DC, Shults CL, et al: Chemical manipulation of hsp70 ATPase activity regulates tau stability. J Neurosci 2009, 29:12079-12088.

10. Dickey CA, Kamal A, Lundgren K, Klosak N, Bailey RM, Dunmore J, Ash P Shoraka S, Zlatkovic J, Eckman CB, et al: The high-affinity HSP90-CHIP complex recognizes and selectively degrades phosphorylated tau client proteins. J Clin Invest 2007, 117:648-658.
11. Dickey CA, Dunmore J, Lu B, Wang JW, Lee WC, Kamal A, Burrows F, Eckman C, Hutton M, Petrucelli L: HSP induction mediates selective clearance of tau phosphorylated at proline-directed Ser/Thr sites but not KXGS (MARK) sites. FASEB J 2006, 20:753-755.

12. Oddo S, Billings L, Kesslak JP, Cribbs DH, LaFerla FM: Abeta immunotherapy leads to clearance of early, but not late, hyperphosphorylated tau aggregates via the proteasome. Neuron 2004, 43:321-332.

13. Wilcock DM, Gharkholonarehe N, Van Nostrand WE, Davis J, Vitek MP, Colton CA: Amyloid reduction by amyloid-beta vaccination also reduces mouse tau pathology and protects from neuron loss in two mouse models of Alzheimer's disease. J Neurosci 2009, 29:7957-7965.

14. Mastrangelo MA, Sudol KL, Narrow WC, Bowers WJ: Interferon-\{gamma\} differentially affects Alzheimer's disease pathologies and induces neurogenesis in triple transgenic-AD mice. Am J Pathol 2009, 175:2076-2088.

15. Zilka N, Stozicka Z, Kovac A, Pilipcinec E, Bugos O, Novak M: Human misfolded truncated tau protein promotes activation of microglia and leukocyte infiltration in the transgenic rat model of tauopathy. $J$ Neuroimmunol 2009, 209:16-25.

16. Kitazawa M, Oddo S, Yamasaki TR, Green KN, LaFerla FM: Lipopolysaccharide-induced inflammation exacerbates tau pathology by a cyclin-dependent kinase 5-mediated pathway in a transgenic model of Alzheimer's disease. J Neurosci 2005, 25:8843-8853.

17. Li Y, Liu L, Barger SW, Griffin WS: Interleukin-1 mediates pathological effects of microglia on tau phosphorylation and on synaptophysin synthesis in cortical neurons through a p38-MAPK pathway. J Neurosci 2003, 23:1605-1611.

18. Cameron B, Landreth GE: Inflammation, microglia, and Alzheimer's disease. Neurobiol Dis 2009, 37:503-509.

19. Colton CA, Mott RT, Sharpe H, Xu Q, Van Nostrand WE, Vitek MP: Expression profiles for macrophage alternative activation genes in $A D$ and in mouse models of AD. J Neuroinflammation 2006, 3:27.

20. Jimenez S, Baglietto-Vargas D, Caballero C, Moreno-Gonzalez I, Torres M, Sanchez-Varo R, Ruano D, Vizuete M, Gutierrez A, Vitorica J: Inflammatory response in the hippocampus of PS1M146L/APP751SL mouse model of Alzheimer's disease: age-dependent switch in the microglial phenotype from alternative to classic. J Neurosci 2008, 28:11650-11661.

21. Quinn J, Montine T, Morrow J, Woodward WR, Kulhanek D, Eckenstein F: Inflammation and cerebral amyloidosis are disconnected in an animal model of Alzheimer's disease. J Neuroimmunol 2003, 137:32-41.

22. Malm TM, Koistinaho M, Parepalo M, Vatanen T, Ooka A, Karlsson S, Koistinaho J: Bone-marrow-derived cells contribute to the recruitment of microglial cells in response to beta-amyloid deposition in APP/PS1 double transgenic Alzheimer mice. Neurobiol Dis 2005, 18:134-142.

23. Herber DL, Mercer M, Roth LM, Symmonds K, Maloney J, Wilson N, Freeman MJ, Morgan D, Gordon MN: Microglial activation is required for Abeta clearance after intracranial injection of lipopolysaccharide in APP transgenic mice. J Neuroimmune Pharmacol 2007, 2:222-231.

24. Herber DL, Roth LM, Wilson D, Wilson N, Mason JE, Morgan D, Gordon MN: Time-dependent reduction in Abeta levels after intracranial LPS administration in APP transgenic mice. Exp Neurol 2004, 190:245-253.

25. DiCarlo G, Wilcock D, Henderson D, Gordon M, Morgan D: Intrahippocampal LPS injections reduce Abeta load in APP+PS1 transgenic mice. Neurobiol Aging 2001, 22:1007-1012.

26. Ray S, Britschgi M, Herbert C, Takeda-Uchimura Y, Boxer A, Blennow K Friedman LF, Galasko DR, Jutel M, Karydas A, et al: Classification and prediction of clinical Alzheimer's diagnosis based on plasma signaling proteins. Nat Med 2007, 13:1359-1362.

27. Spires TL, Orne JD, SantaCruz K, Pitstick R, Carlson GA, Ashe KH, Hyman BT: Region-specific dissociation of neuronal loss and neurofibrillary pathology in a mouse model of tauopathy. Am J Pathol 2006, 168:1598-1607.

28. Gordon MN, Holcomb LA, Jantzen PT, DiCarlo G, Wilcock D, Boyett KW, Connor K, Melachrino J, O'Callaghan JP, Morgan D: Time course of the development of Alzheimer-like pathology in the doubly transgenic PS1 +APP mouse. Exp Neurol 2002, 173:183-195.

29. Morris SM Jr, Kepka-Lenhart D, Chen LC: Differential regulation of arginases and inducible nitric oxide synthase in murine macrophage cells. Am J Physiol 1998, 275:E740-747. 
30. Dickey C, Kraft C, Jinwal U, Koren J, Johnson A, Anderson L, Lebson L, Lee $D$, Dickson $D$, de Silva $R$, et al: Aging analysis reveals slowed tau turnover and enhanced stress response in a mouse model of tauopathy. Am J Pathol 2009, 174:228-238.

31. Rosi S, Ramirez-Amaya V, Vazdarjanova A, Worley PF, Barnes CA, Wenk GL: Neuroinflammation alters the hippocampal pattern of behaviorally induced Arc expression. J Neurosci 2005, 25:723-731.

32. Hung Sl, Chang AC, Kato I, Chang NC: Transient expression of Ym1, a heparin-binding lectin, during developmental hematopoiesis and inflammation. J Leukoc Biol 2002, 72:72-82.

33. Martinez FO, Helming L, Gordon S: Alternative activation of macrophages: an immunologic functional perspective. Annu Rev Immunol 2009, 27:451-483.

34. Sun YJ, Chang NC, Hung SI, Chang AC, Chou CC, Hsiao CD: The crystal structure of a novel mammalian lectin, Ym1, suggests a saccharide binding site. J Biol Chem 2001, 276:17507-17514.

35. Goedert M, Jakes R, Spillantini MG, Hasegawa M, Smith MJ, Crowther RA: Assembly of microtubule-associated protein tau into Alzheimer-like filaments induced by sulphated glycosaminoglycans. Nature 1996, 383:550-553.

36. Janelsins MC, Mastrangelo MA, Park KM, Sudol KL, Narrow WC, Oddo S, LaFerla FM, Callahan LM, Federoff HJ, Bowers WJ: Chronic neuron-specific tumor necrosis factor-alpha expression enhances the local inflammatory environment ultimately leading to neuronal death in $3 \times \mathrm{Tg}-\mathrm{AD}$ mice. Am J Pathol 2008, 173:1768-1782.

37. McKee AC, Carreras I, Hossain L, Ryu H, Klein WL, Oddo S, LaFerla FM, Jenkins BG, Kowall NW, Dedeoglu A: Ibuprofen reduces Abeta, hyperphosphorylated tau and memory deficits in Alzheimer mice. Brain Res 2008, 1207:225-236.

38. Shaftel SS, Kyrkanides S, Olschowka JA, Miller JN, Johnson RE, O'Banion MK: Sustained hippocampal IL-1 beta overexpression mediates chronic neuroinflammation and ameliorates Alzheimer plaque pathology. I Clin Invest 2007, 117:1595-1604.

39. Chakrabarty $P$, Jansen-West $K$, Beccard A, Ceballos-Diaz C, Levites $Y$, Verbeeck C, Zubair AC, Dickson D, Golde TE, Das P: Massive gliosis induced by interleukin- 6 suppresses Abeta deposition in vivo: evidence against inflammation as a driving force for amyloid deposition. FASEB J 2009, 24:548-559.

doi:10.1186/1742-2094-7-56

Cite this article as: Lee et al: LPS- induced inflammation exacerbates phospho-tau pathology in rTg4510 mice. Journal of Neuroinflammation 2010 7:56.

\section{Submit your next manuscript to BioMed Central and take full advantage of:}

- Convenient online submission

- Thorough peer review

- No space constraints or color figure charges

- Immediate publication on acceptance

- Inclusion in PubMed, CAS, Scopus and Google Scholar

- Research which is freely available for redistribution

Submit your manuscript at www.biomedcentral.com/submit
Biomed Central 